
\title{
3 Research Square \\ Kallistatin in Follicular Fluid of Women with \\ Endometriosis and Its Correlation with IVF Outcome
}

\section{Yuling Mao}

Third Affiliated Hospital of Guangzhou Medical College https://orcid.org/0000-0002-9931-6579

\section{Shaoquan Zhan}

Third Affiliated Hospital of Guangzhou Medical College

\section{Jingda Qiao}

Institute of Neuroscience and Department of Neurology of the Second Affiliated Hospital of Guangzhou

Medical University

Lei Li

Third Affiliated Hospital of Guangzhou Medical College

Hanyan Liu

Third Affiliated Hospital of Guangzhou Medical College

Rui Chen ( $\square$ ruich@126.com )

Third Affiliated Hospital of Guangzhou Medical College

Research

Keywords: Endometriosis; Kallistatin; follicular fluid; IVF outcome

Posted Date: January 9th, 2021

DOl: https://doi.org/10.21203/rs.3.rs-140378/v1

License: (c) (i) This work is licensed under a Creative Commons Attribution 4.0 International License.

Read Full License

Version of Record: A version of this preprint was published at Gynecological Endocrinology on July 8th, 2021. See the published version at https://doi.org/10.1080/09513590.2021.1928067. 


\section{Abstract}

Background: Endometriosis (EM) affects $10 \%$ of women in reproductive age and alters fertility. Its management is still debated notably the timing of surgery and ART in infertility. Kallistatin (KS) is an endogenous protein that regulates differential signaling pathways and biological functions. However, the function and the underlying molecular mechanism in endometriosis and its correlation with IVF outcome have not been determined. The purpose of this study was to evaluate KS concentrations in follicular fluid of women with EM and controls women without EM who underwent IVF with embryo transfer (IVF-ET).

Methods: Follicular fluid KS concentrations from 40 patients with EM and 40 non-EM patients were measured by ELISA.

Results: Compared with the non-EM patients, patients with EM had lower KS levels in follicular fluid $(281.67 \pm 104.60 \mathrm{pg} / \mathrm{ml} v s .490 .70 \pm 216.33 \mathrm{pg} / \mathrm{ml})$. The rates of fertilization $(61.64 \pm 22.42 \% v s$. $71.00 \pm 24.39 \%)$, available embryo $(45.96 \pm 19.83 \% v s .50 .61 \pm 26.26 \%)$ and top-quality embryo(12.71 $\pm 21.01 \%$ vs.16.04 $\pm 16.87 \%)$ were significantly lower in the EM group than in the control group. The KS concentrations in the follicular fluid of women who conceived consequent to the treatment were significantly higher than those from women who did not in the combined EM and control groups.

\section{Conclusions:}

The present results indicate that the KS concentration in follicular fluid could be used as a predictor for IVF-ET outcomes. This may contribute to the pathologic mechanism responsible for the poor outcome of IVF in patients with EM.

\section{Background}

Endometriosis is a common chronic gynecologic disorder defined by the ectopic occurrence of endometrium-like tissue which is associated with pelvic pain and infertility[1]. It has been estimated at $10 \%$ in women of reproductive age[2]. The causes of infertility in women with endometriosis may range from anatomical distortions due to adhesions and fibrosis to abnormalities and immunological disturbances[3, 4]. Approximately $25-50 \%$ of infertile women have EM, and $30-50 \%$ of women with EM are infertile[5]. Assisted reproductive technology (ART) may be required with endometriosis-related infertility. Accumulating evidence suggests that EM affects oocyte and embryo development as well as endometrial receptivity, resulting in poor in vitro fertilization (IVF) outcomes[6-10]. However, the mechanism is unclear.

Follicular fuid (FF) provides an environment that controls oocyte growth and maturation, and plays a key role in ovulation, subsequent fertilization and early embryo development[11]. Both cellular and secreted mediators are aberrantly expressed in the peritoneal fuid (PF) and plasma of EM patients[12]. Some studies have shown that angiogenesis and tissue growth factor are increased in the serum and PF of women with $\mathrm{EM}[13,14]$. 
For these reasons, we analyzed the concentrations of KS in the FF of women undergoing IVF to identify factors related to EM and to further elucidate its correlation with IVF outcome. KS is an endogenous protein that regulates differential signaling pathways and biological functions inhibiting inflammation, angiogenesis, oxidative stress, apoptosis, tumor growth and metastasis in animal models and cultured cells[15-22]. Plasma levels of KS are reduced in patients with sepsis, liver disease and obesity[23, 24]. Our preliminary data showed that KS in EM patients' endometrial tissue and blood were much lower than control patients without endometriosis and the decrease of KS is correlated with the severity of endometriosis. However, the concentrations of KS in the follicular fluid of EM patients and its correlation with IVF outcome have not been determined.

The aim of this study was to investigate KS in follicular fluid of women with endometriosis and its correlation with IVF outcome. This information may form the basis of strategies to control these factors and improve the IVF outcomes in patients with EM.

\section{Methods}

\section{Subjects}

A total of 80 patients who underwent IVF-ET between July 2017 and August 2018 in the Center for Reproductive Medicine, Third Affiliated Hospital of Guangzhou Medical University, were recruited for this study. The study population consisted of Forty women with EM and 40 women without EM. The presence or absence of endometriosis was confirmed by laparoscopic surgery and post-operative histological examination. Women with polycystic ovary syndrome, diabetes, hypertension, dyslipidemia, HIV infection or any active infection and autoimmune diseases were excluded. Follicular fluid (FF) was extracted from both groups.

\section{ART procedures}

Women were monitored and managed according to the hospital's clinical protocols. Various controlled ovarian stimulation (COS) protocols were used, with 150-450 IU/day of recombinant FSH or human menopausal gonadotropin in a gonadotropin-releasing hormone antagonist protocol, a long agonist protocol, or a short agonist protocol. The protocols were determined according to each patient's characteristics (age, body mass index (BMI), AFC and AMH). Transvaginal oocyte retrieval was scheduled 35-36 $\mathrm{h}$ after $\mathrm{hCG}$ injection ART was performed per standard operating procedure of the hospital. Fertilization was assessed by the appearance of two pronuclei. Cleavage stage embryos were graded as per the Istanbul consensus. Fresh embryo transfer (ET) was performed 2-3 or 5 days later. Embryos were vitrified frozen on day 3,5 or 6 . The luteal phase was supported by vaginal administration of micronized progesterone $(400 \mathrm{mg} / \mathrm{d})$ started on the day of ovarian puncture.

\section{Follicular fluid collection}


Follicular fluid was preserved at oocyte retrieval, by collecting the liquid aspirated from the follicle into the suction tube, to avoid contamination by blood. Follicular fluid samples from each follicle were pooled for each patient for measurement of KS concentrations. Pooled follicular fluid was centrifuged at $1500 \mathrm{~g}$ for $10 \mathrm{~min}$ to eliminate cells and cell debris. And the supernatants were then stored at $-20^{\circ} \mathrm{C}$ for $\mathrm{KS}$ concentration measurements.

\section{KS and hormone assays}

The follicular fluid KS concentrations were collected and determined measured by enzyme-linked immunosorbent assay (human kallistatin ELISA kits, Catalog Number: DY1669; R\&D Systems, USA) according to the manufacturer's protocol. The concentrations of serum oestradiol, FSH, LH, progesterone, testosterone and $\mathrm{AMH}$, and follicular fluid oestradiol and progesterone, were measured by chemiluminescence (Roche, Switzerland).

\section{Outcome measure}

The reproductive outcome of this study included implantation, clinical pregnancy, live birth rate (LBR) and spontaneous miscarriage rates. Neonatal outcomes included preterm birth, stillbirth, birth weight, low birth weight and congenital anomalies. Live birth was defined as the delivery of any viable neonate who was 28 weeks of gestation or older, and twins delivered by one mother were calculated as one live birth. Clinical pregnancy was defined as the present of gestational sac on ultrasound at 6-8 weeks of gestation; low birth weight was defined as the birth weight less than $2500 \mathrm{~g}$ and very low birth weight less than $1500 \mathrm{~g}$.

\section{Statistical analysis}

The statistical analysis was performed using the Statistical Package for Social Science (SPSS) version 22.0. The baseline characteristic was expressed as the mean \pm SD (standard deviation) and differences in variables were compared by means of Student's t-test. Categorical variables were described as frequencies and percentages, and compared using chi-square test. A $P$-value of 0.05 was considered significant.

\section{Results}

\section{Patient characteristics}

Eighty women were selected. Baseline characteristics of these women are shown in Table 1. No significant differences were observed between the EM and control groups regarding age, body mass index and duration of sterility. In the control group, there were 4 patients with infertility due to tubal factor $(10 \%)$, two cases of ovulatory dysfunction (5\%), 20 cases of male factor $(50 \%), 14$ cases of unexplained (35\%).30 patients in the control group were given IVF protocols, while 10 underwent ICSI procedures. In EM group, IVF protocols were given to $70 \%$ women and $30 \%$ underwent ICSI procedures. 


\section{KS expression was decreased in FF of EM patients}

Serum concentration of base $\mathrm{LH}, \mathrm{FSH}$, Progesterone(P), Testosterone $(\mathrm{T}), \mathrm{E} 2$ and $\mathrm{AMH}$ showed no difference between the EM and control groups. Moreover, The FF KS concentrations in the EM patients were $281.67 \pm 104.60 \mathrm{pg} / \mathrm{ml}$ and lower than those in the control group $(490.70 \pm 216.33 \mathrm{pg} / \mathrm{ml})(P<0.05)$ (Table 2).

\section{ART Cycle Characteristics in EM and control subjects.}

Comparisons of ART Cycle Characteristics between the EM and control groups were summarized in Table 3. The AFC, Dosage of Gn, Endometrial thickness, No. of embryos retrieved, the rates of cleavage and No. of embryos transferred were similar among between the two FET groups. Meanwhile, the rates of fertilization, available embryo and top-quality embryo were significantly lower in the EM group than in the control group.

\section{KS concentrations in follicular fluid from subjects with different pregnancy outcomes}

The clinical pregnancy rate in the EM group was $61.54 \%$ (24/39), which was significantly lower than that in the control group $(67.57 \%, 25 / 37)$. In both the EM (Figure 1B) and control groups (Figure 1A), the KS concentrations in the follicular fluid of women who conceived consequent to the treatment were significantly higher than those in women who did not. The KS concentrations in the follicular fluid of women who conceived consequent to the treatment were significantly higher than those from women who did not in the combined EM and control groups $(P<0.05$; Figure $1 \mathrm{C})$. To evaluate the potential of KS concentrations in the follicular fluid for predicting clinical pregnancy, receiver operating characteristic (ROC) curves were plotted, and the area under the curve (AUC) values were determined with the highest specificity and sensitivity set as the optimal prediction point (Figure 1D). Optimal prediction point, sensitivities, specificities and AUC values were $292.78 \mathrm{pg} / \mathrm{ml}, 71.7 \%, 65 \%$ and 0.7279 , respectively. The present results indicate that the KS concentration in follicular fluid could be used as a predictor for IVFET outcomes.

\section{Discussion}

FF composition is complex and is basically derived from plasma exudation and ovarian secretion[25]. It is an important environment for oocyte survival and directly affects oocyte maturation and quality. FF contains a large number of cytokines, a variety of hormones, oxidation/ antioxidant systems and metabolic products [13].

In this study, analysis of the levels of KS in follicular fluid of women with EM and controls women without EM who underwent IVF with embryo transfer (IVF-ET). Data clearly show that $\$ compared with the non-EM patients, patients with EM had lower KS levels in follicular fluid. This indicates that low KS concentrations in follicular fluid may play a key role in folliculogenesis and the pathogenesis of EM. However, there were no differences in the concentrations of $\mathrm{LH} \square \mathrm{FSH} \square \mathrm{P} \square T \mathrm{~T} 2$ 2 and AMH between the EM and control groups. 
This means that the KS changes of follicular microenvironment caused by EM are independent of these six factors.

$\mathrm{KS}$ is an endogenous protein that regulates differential signaling pathways and biological functions. Our preliminary data showed that KS in EM patients' endometrial tissue and blood were much lower than control patients without endometriosis and the decrease of KS is correlated with the severity of endometriosis. However, the concentrations of KS in the follicular fluid of EM patients and its correlation with IVF outcome have not been determined. This study found that the rates of fertilization, available embryo and top-quality embryo were significantly lower in the EM group than in the control group. This result is consistent with the worse prognosis of EM reported in previous literature. We supposed that KS can significantly improve the quality of oocytes and embryos.

However, whether KS can act as a biomarker for predicting the clinical pregnancy of EM remain unknown. ROC curves were plotted in our study, result showed that the area under the curve (AUC) values was 0.7279. The present results indicate that the KS concentration in follicular fluid could be used as a predictor for IVF-ET outcomes.

Based on the findings of our study, we conclude that KS is associated with the microenvironment of FF in patients with EM. The change in the regulation network in EM influence oocyte development, resulting in poor IVF outcomes. However, the results in this study should be validated in a large sample in the future.

\section{Conclusion}

Our study provides a theoretical basis for the development of strategies to improve the outcome of IVF in patients with EM. This evidence may help to clarify the pathology of EM and seek new treatments for EM patients.

\section{Abbreviations}

Endometriosis

Kallistatin

in vitro fertilization

peritoneal fuid

Follicular fuid

controlled ovarian stimulation

body mass index

BMI

EM

KS

IVF

PF

FF live birth rate

LBR

cos 
area under the curve $\quad$ AUC

luteinizing hormone $\quad \mathrm{LH}$

Follicle-Stimulating Hormone FSH

progesterone $\quad \mathrm{P}$

testosterone $\quad T$

estrogen $\quad E 2$

Anti-mullerian hormone $\quad \mathrm{AMH}$

\section{Declarations}

\section{Ethical Approval and Consent to participate}

This study was approved by the ethics committee of the Third Affiliated Hospital of Guangzhou Medical University.

\section{Consent for publication}

Not applicable.

\section{Availability of supporting data}

The data sets used and/or analyzed during the current study are available from the corresponding author on reasonable request.

\section{Competing interests}

The authors have declared that no conflict of interest exists.

\section{Funding}

This study was supported by National Nature Science Foundation of China, Grant Numbers: 81871211 Authors' contributions

All authors have contributed significantly to this manuscript and each author has contributed to the manuscript as follows: Yuling Mao and Rui Chen designed the project. Yuling Mao, Shaoquan Zhan collected clinical samples and performed the ELISA experiments. Jingda Qiao and Lei Li analyzed the data. Yuling Mao wrote the manuscript. Hanyan Liu and Rui Chen revised the manuscript. All authors 
read and approved the final manuscript.

Acknowledgements

The study was performed under the auspices of the IVF unit of the Third Affiliated Hospital of Guangzhou Medical University.

\section{References}

1. Gordts S, Koninckx P, Brosens I. Pathogenesis of deep endometriosis. Fertil Steril. 2017;108(6):87285 e1.

2. Meuleman C, et al. High prevalence of endometriosis in infertile women with normal ovulation and normospermic partners. Fertil Steril. 2009;92(1):68-74.

3. Senapati S, et al. Impact of endometriosis on in vitro fertilization outcomes: an evaluation of the Society for Assisted Reproductive Technologies Database. Fertil Steril. 2016;106(1):164-71 e1.

4. Vercellini P, et al. Endometriosis: pathogenesis and treatment. Nat Rev Endocrinol. 2014;10(5):26175 .

5. Du YB, et al. Endocrine and inflammatory factors and endometriosis-associated infertility in assisted reproduction techniques. Arch Gynecol Obstet. 2013;287(1):123-30.

6. Da Broi MG, et al. Oocyte oxidative DNA damage may be involved in minimal/mild endometriosisrelated infertility. Mol Reprod Dev. 2018;85(2):128-36.

7. Garrido N, et al. The endometrium versus embryonic quality in endometriosis-related infertility. Hum Reprod Update. 2002;8(1):95-103.

8. Barnhart K, Dunsmoor-Su R, Coutifaris C. Effect of endometriosis on in vitro fertilization. Fertil Steril. 2002;77(6):1148-55.

9. Freis A, et al. Relative Morphokinetics Assessed by Time-Lapse Imaging Are Altered in Embryos From Patients With Endometriosis. Reprod Sci. 2018;25(8):1279-85.

10. Sanchez AM, et al., Is the oocyte quality affected by endometriosis? A review of the literature. Journal of Ovarian Research, 2017. 10.

11. Da Broi MG, et al. Influence of follicular fluid and cumulus cells on oocyte quality: clinical implications. J Assist Reprod Genet. 2018;35(5):735-51.

12. Miller JE, et al. Implications of immune dysfunction on endometriosis associated infertility. Oncotarget. 2017;8(4):7138-47.

13. Taylor RN, Lebovic DI, Mueller MD, Angiogenic factors in endometriosis. Ann N Y Acad Sci, 2002. 955: p. 89-100; discussion 118, 396-406.

14. Deguchi M, et al. Expression of angiogenic factors in extrapelvic endometriosis. Oncol Rep. 2001;8(6):1317-9.

15. Chao J, et al. Kallistatin, a novel human tissue kallikrein inhibitor: levels in body fluids, blood cells, and tissues in health and disease. J Lab Clin Med. 1996;127(6):612-20. 
16. Zhu H, et al. Plasma kallistatin is associated with adiposity and cardiometabolic risk in apparently healthy African American adolescents. Metabolism. 2013;62(5):642-6.

17. Miao RQ, et al. Structural elements of kallistatin required for inhibition of angiogenesis. Am J Physiol Cell Physiol. 2003;284(6):C1604-13.

18. Shen B, et al. Kallistatin attenuates endothelial apoptosis through inhibition of oxidative stress and activation of Akt-eNOS signaling. Am J Physiol Heart Circ Physiol. 2010;299(5):H1419-27.

19. Shen B, et al. Salutary effect of kallistatin in salt-induced renal injury, inflammation, and fibrosis via antioxidative stress. Hypertension. 2008;51(5):1358-65.

20. Wang CR, et al. Prophylactic adenovirus-mediated human kallistatin gene therapy suppresses rat arthritis by inhibiting angiogenesis and inflammation. Arthritis Rheum. 2005;52(4):1319-24.

21. Yin $\mathrm{H}$, et al. Kallistatin inhibits vascular inflammation by antagonizing tumor necrosis factor-alphainduced nuclear factor kappaB activation. Hypertension. 2010;56(2):260-7.

22. Shiau $A L$, et al. Inhibition of experimental lung metastasis by systemic lentiviral delivery of kallistatin. BMC Cancer. 2010;10:245.

23. Gao L, et al. Role of kallistatin in prevention of cardiac remodeling after chronic myocardial infarction. Lab Invest. 2008;88(11):1157-66.

24. Miao RQ, et al. Kallistatin is a new inhibitor of angiogenesis and tumor growth. Blood. 2002;100(9):3245-52.

25. Kollmann Z, et al. Gonadotrophin stimulation in IVF alters the immune cell profile in follicular fluid and the cytokine concentrations in follicular fluid and serum. Hum Reprod. 2017;32(4):820-31.

\section{Tables}

Due to technical limitations, table 1 to 3 is only available as a download in the Supplemental Files section.

\section{Figures}


A

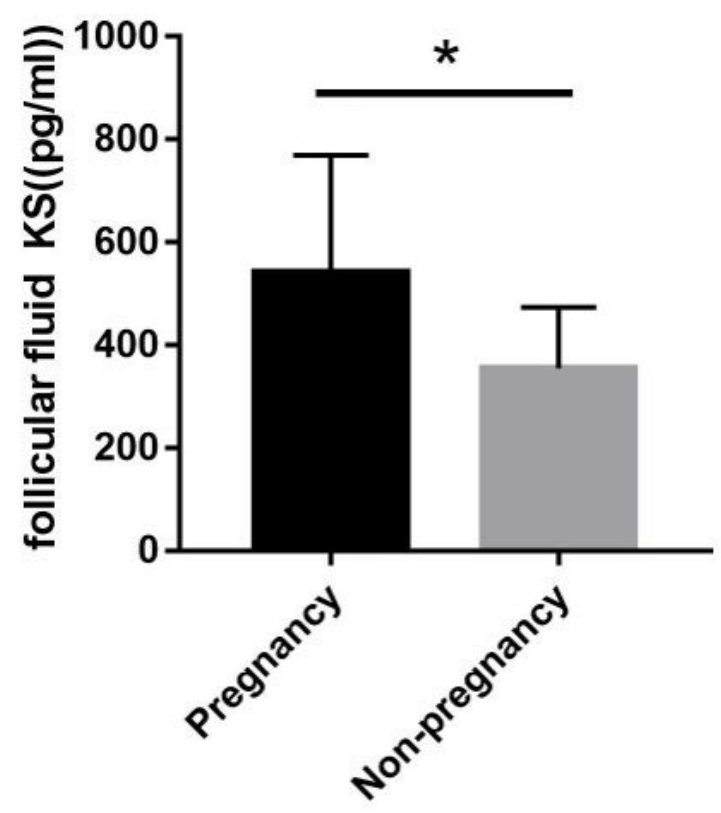

C

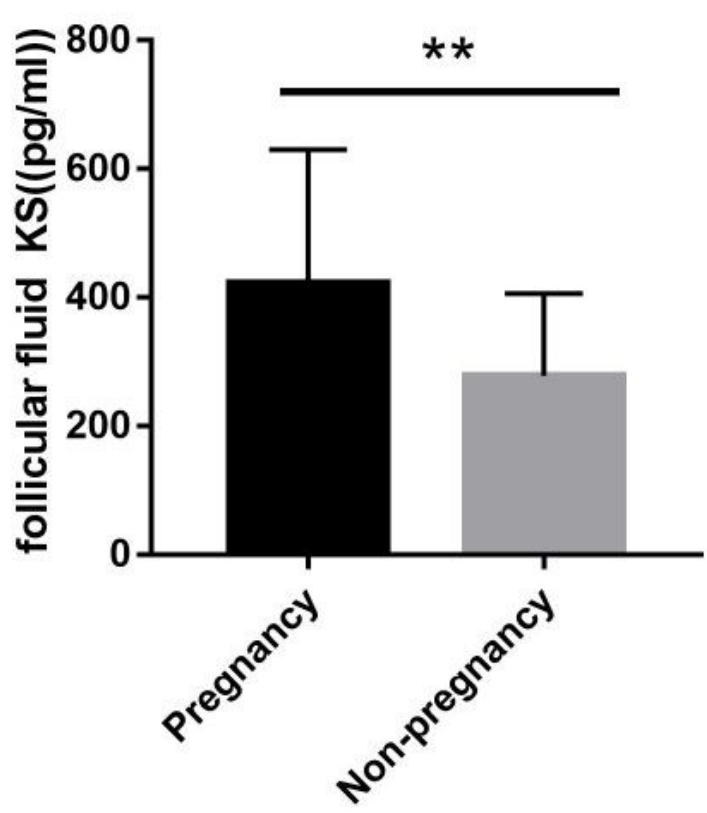

B

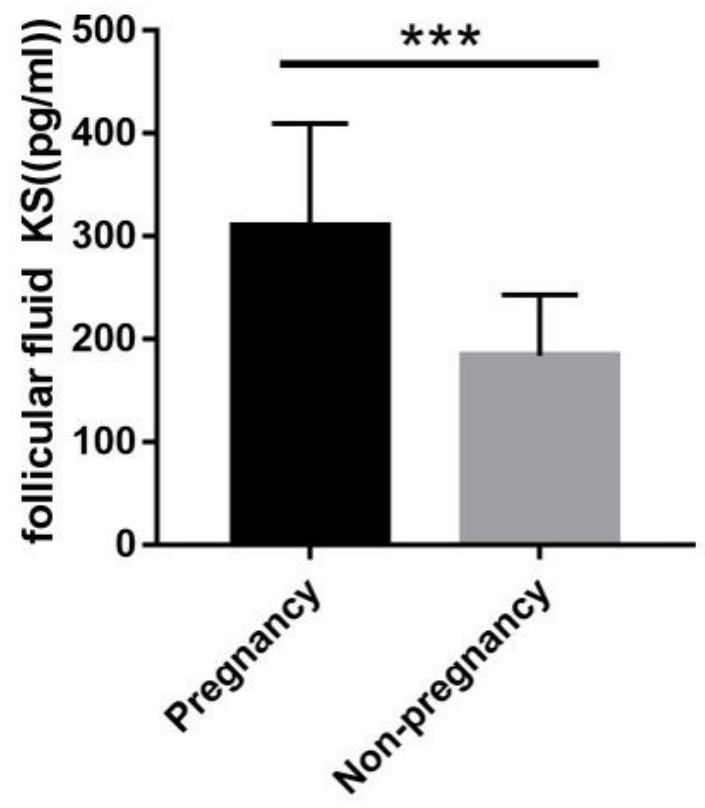

D

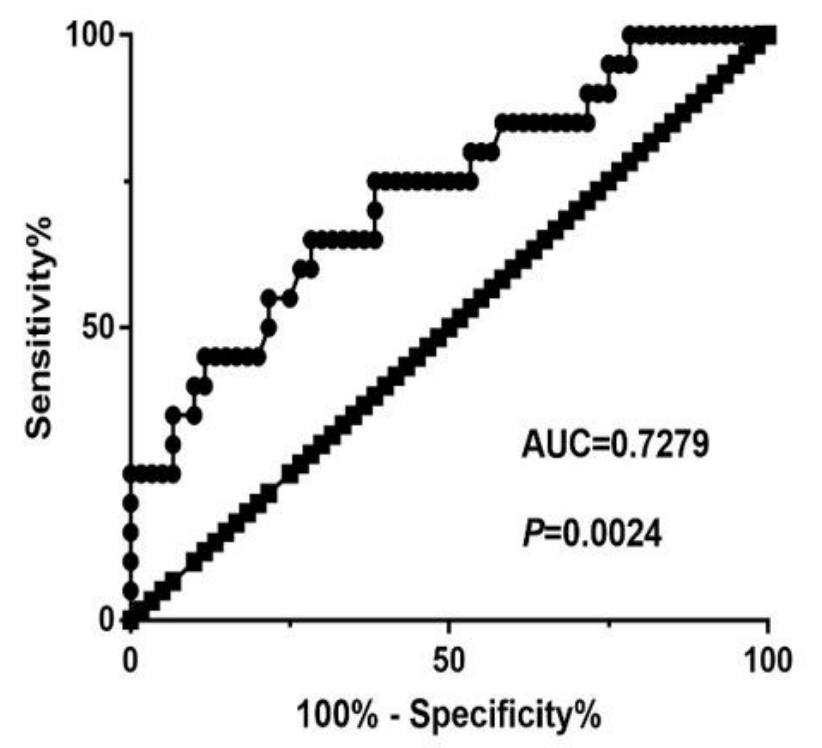

Figure 1

KS concentrations in women who conceived consequent to the treatment (Pregnancy) and women who did not (Non-pregnancy). (A) KS concentrations in follicular fluid of control women in women who conceived consequent to the treatment and those who did not. (B) KS concentrations in follicular fluid of EM patients who conceived consequent to the treatment and those who did not. (C) KS concentrations in follicular fluid of all women who conceived consequent to the treatment and women who did not. $\left({ }^{\star} \mathrm{P}<\right.$ 0.05). (D) ROC curve analysis to assess the predictive powers of clinical pregnancy. 


\section{Supplementary Files}

This is a list of supplementary files associated with this preprint. Click to download.

- table1.jpg

- table2.jpg

- table3.jpg 